\title{
Efecto de las condiciones de síntesis y purificación sobre la estabilidad de fenil-amino-dietoxi-silanos
}

\author{
Alejandro Ramírez VÉlez ${ }^{1}$ \\ DANIEL ESTEBAN JARAMILLO ${ }^{1}$ \\ LUISA FERNANDA TABARES ${ }^{1}$ \\ MÓNICA MESA CADAVID ${ }^{1}$
}

\section{Resumen}

Los organil-amino-alcoxi-silanos son moléculas interesantes para la síntesis de materiales híbridos. Su uso se ha visto limitado debido a la escasa oferta comercial y a su baja estabilidad. En este trabajo se prepararon tres fenil-aminodietoxi-silanos mediante condensación de fenil-cloro-dietoxi-silano y aminas (ruta 1) y fenil-trietoxi-silano y amiduros de magnesio (ruta 2). La caracterización y los estudios de estabilidad de los compuestos fueron llevados a cabo por ${ }^{1} \mathrm{H}-\mathrm{RMN}$ y CG-MS. Ambas rutas presentaron rendimientos mayores a 91\%. Fue posible establecer que la sal acida, obtenida en la ruta 1, cataliza la descomposición total de los productos en un tiempo inferior a dos semanas. El medio de reacción de la ruta 2 mostró ser mucho menos agresivo, y después de dos semanas, aún permanece más del 60\% de la concentración inicial.

Palabras clave: Amiduros de magnesio; amino-silanos; cloro-silanos; silanos heterofuncionales; materiales híbridos; polisiloxanos

\section{Purification and synthesis conditions effect on the phenyl-amino-diethoxy-silanes stability}

\section{Abstract}

Organyl-amino-alkoxy-silanes are interesting molecules for the synthesis of hybrid materials. Due to a scarce commercial availability and a low stability, their use has been limited. In this work, three phenyl-amino-diethoxy-silanes

1 Universidad de Antioquia, U. de A. Medellín, Colombia.

Autor de correspondencia: Ramírez Vélez (Alejandro): Instituto de Química, Universidad de Antioquia, A.A 1226. Medellín, Colombia..

Correo electrónico: alejandro.ramirez@udea.edu.co
Historia del artículo:

Artículo recibido: 21-IX-2018/ Aprobado: 30-V-2019

Disponible online: 3 de julio de 2019

Discusión abierta hasta marzo de 2021 
were prepared by the condensation of phenyl-chloro-diethoxy-silane and amines (route 1) and phenyl-triethoxy-silane and magnesium amides (route 2). The characterization and the stability studies of the compounds were carried out by ${ }^{1} \mathrm{H}-\mathrm{NMR}$ and GC-MS. Both routes showed yields greater than $91 \%$. It was possible to establish that the acid salt obtained in route 1 , catalyzes the total decomposition of the products in less than two weeks. The reaction medium of route 2 was much less aggressive and after two weeks, more than $60 \%$ of the initial concentration still remains.

Keywords: Magnesium amides; amino-silanes; Chloro-silanes; heterofunctional silanes; hybrid materials; polysiloxanes.

\section{Efeito das condições de síntese e purificação na estabilidade de fenil-amino-dietoxi-silanos}

\section{Resumo}

Os organil-amino-alcoxi-silanos são moléculas interessantes para a sínteses desses materiais híbridos. 0 seu uso se tem visto limitado devido à escassa oferta comercial e a sua baixa estabilidade. Neste trabalho se prepararam três fenil-amino-dietoxi-silanos por meio da condensação de fenil-cloro-dietoxi-silano e aminas (rota 1) e fenil-trietoxi-silano e amiduros de magnésio (rota 2). A caracterização e os estudos de estabilidade dos compostos foram levados a bom fim por ${ }^{1} \mathrm{H}-\mathrm{RMN}$ e CG-MS. Ambas rotas presentaram rendimentos superiores ao 91\%. Foi possível estabelecer que o sal ácido, obtido na ruta 1, catalisa a decomposição total dos produtos numtempo inferior as duas semanas. 0 médio de reação da rota 2 mostrou ser muito menos agressivo, e depois de dois semanas, ainda permaneceu mais do $60 \%$ da concentração inicial.

Palavras-chave: Amiduros de magnésio; amino-silanos; cloro-silanos; silanos heterofuncionales; materiais híbridos; polissiloxanos.

\section{Introducción}

La policondensación hidrolítica de silanos homofuncionales con formula $\mathrm{PhSiX}_{3}(\mathrm{Ph}=$ fenil, $\mathrm{X}$ = grupo funcional hidrolizable como $\mathrm{H}$, halógeno, $\mathrm{OCH}_{2} \mathrm{CH}_{3}$ o $\mathrm{OCH}_{3}$ ) es utilizada para la síntesis de diferentes tipos de materiales híbridos (Abe y Gunji, 2004). Dependiendo de las condiciones bajo las cuales se lleven a cabo las reacciones de hidrólisis y condensación de los grupos X (Figura 1, mecanismo A), es posible preparar polímeros capaces de formar fibras o películas flexibles, partículas con estructura atómica aleatoria similar a la de las sílices, nanopartículas con estructuras tridimensionales ordenadas como los silsesquioxanos oligoméricos poliédricos (SSOP), entre otros. Debido a su naturaleza quími- ca semejante, los grupos X hidrolizan simultáneamente y condensan de forma no selectiva. A este comportamiento se le atribuye el hecho de que en muchos casos, el material buscado se obtenga con bajos rendimientos y en presencia de una mezcla de subproductos no deseados difíciles de separar. Por otro lado, la síntesis de materiales a partir de silanos heterofuncionales con formula $\mathrm{PhSiX}_{2} \mathrm{Y}$ (siendo Y un grupo reactivo diferente de $\mathrm{X}$ ) como los fenil-aminodialcoxi-silanos $\mathrm{PhSi}(\mathrm{OR})_{2} \mathrm{NR}_{1} \mathrm{R}_{2}\left(\mathrm{R}=\mathrm{CH}_{3} \mathrm{O} \mathrm{CH}_{2} \mathrm{CH}_{3}\right.$, $\mathrm{R}_{1}, \mathrm{R}_{2}=$ alquil o fenil) o los difenil-diamino-dialcoxidisiloxanos (ver compuesto (9) de la Figura 1), ha sido poco explorada. La presencia de enlaces Si-O y Si-N, los cuales pueden hidrolizar de manera sucesiva en lugar de simultánea, permitiría en teoría 
dirigir la policondensación de forma más eficiente y selectiva (ver Figura 1, mecanismo B). Debido a su escasa oferta comercial, si se desean utilizar estos silanos heterofuncionales como bloques de construcción para la preparación de materiales, es necesario primero sintetizarlos en el laboratorio.

Figura 1. Productos obtenidos a partir de hidrólisis y condensación simultanea de silanos homofuncionales (mecanismo A) e hidrólisis y condensación dirigida de silanos heterofuncionales (mecanismo B). (3) diorganil-disiloxano-tetraol, (4) oligosiloxano lineal, (5) tetraorganil-ciclosiloxano-tetraol, (6) silsesquioxano oligomérico poliédrico (SSOP), (7) silsesquioxano aleatorio (resina de silicona), (8) silsesquioxano tipo escalera, (9) difenil-diamino-dialcoxi-disiloxanos

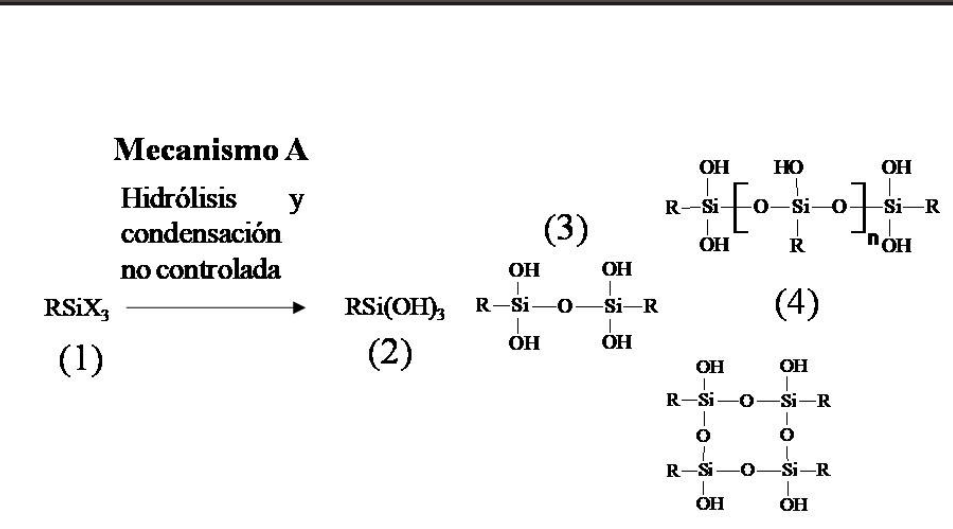

(5)

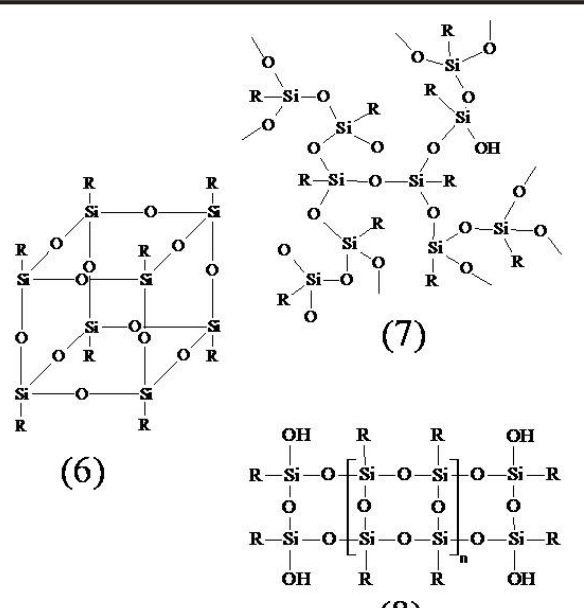

(8)

Grado de condensación

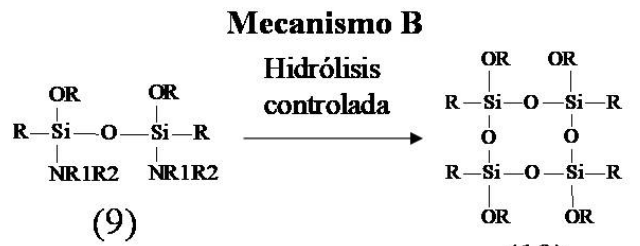

(10)

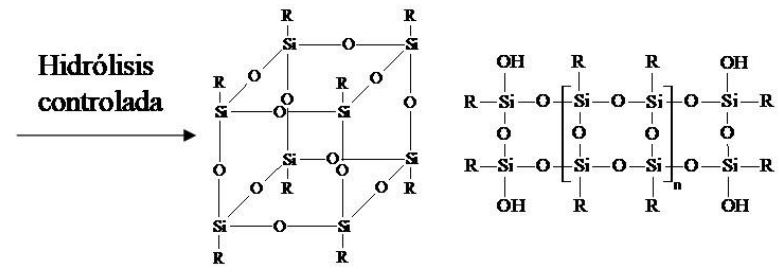

Figura 2. Estrategias de síntesis hacia organil-amino-dialcoxi-silanos a partir de organil-trialcoxi-silanos 
Los amino-alcoxi-silanos son tradicionalmente sintetizados a partir de alcoxi-silanos mediante su cloración y aminación (ruta 1 de la Figura 2). También se pueden preparar mediante reacción directa con amiduros de litio (Bauer y Strohmann, 2012; Spence et al., 2013) o bases de amiduros de magnesio (ruta 2 de la Figura 2). En ambas rutas se obtiene un subproducto sólido, por lo que la purificación involucra filtración y posterior destilación al vacío. Una breve descripción de las publicaciones más representativas relacionadas con la ruta $1 \mathrm{y}$ con los amiduros de magnesio de la ruta 2 será presentada a continuación.

La condensación de cloro-silanos y aminas primarias, secundarias o amoniaco, la cual procede con la eliminación de cantidades estequiométricas de $\mathrm{HCl}$, es sin lugar a dudas la ruta más utilizada (Marciniec et al., 2015). La aminación de organilcloro-silanos es utilizada con frecuencia en síntesis orgánica como protocolo temporal de protección de aminas (Colvin, 1990; Ishibashi et al., 2006). También en cromatografía de gases para la preparación de derivados (Molnár et al., 2015; Drozd, 1981). Sin embargo, es importante notar que además del cloro, los silanos utilizados en esos casos (e.g trimetil-cloro-silano, $t$-butil-difenil-cloro-silano, dimetil-fenilcloro-silano) no poseen otros grupos hidrolizables como en el caso de los organil-dialcoxi-cloro-silanos. Como estudios pioneros de la ruta 1 podemos mencionar la síntesis en dos etapas (Miner et al., 1947) y las reacciones entre trialcoxi-cloro-silanos y amoniaco o dimetilamina (Rosnati, 1948). Miner preparó en una primera etapa, $t$-alcoxi-cloro-silanos a partir de la reacción de $\mathrm{SiCl}_{4}$ y alcoholes terciarios, usando piridina como catalizador básico. Posteriormente el cloro remanente fue aminado haciendo pasar amoniaco bajo condiciones de temperatura controlada. Los autores observaron una pobre estabilidad de sus productos, los cuales hidrolizaron rápidamente originando un material resinoso hidrofóbico. Rosnati encontró que con el uso de $\mathrm{NH}_{3}$ se producian disilazanos en lugar de los amino-trialcoxi-silanos y que la destilación de estos derivados a presión atmosférica originaba el desprendimiento de amoniaco y la formación de $\mathrm{SiO}_{2}$. Por otro lado, él logró preparar dimetilamino-trietoxi-silano haciendo pasar vapores de $\left(\mathrm{CH}_{3}\right)_{2} \mathrm{NH}$ a través de una mezcla de benceno y trietoxi-cloro-silano. Esta reacción fue acompañada por la aparición de la sal $\left(\mathrm{CH}_{3}\right)_{2} \mathrm{NH}_{2} \mathrm{Cl}$, originada por neutralización de parte de la dimetilamina con el $\mathrm{HCl}$ formado. Para purificar el producto, la sal fue removida por filtración y el filtrado sometido a destilación al vacío. Rosnati pudo comprobar que, en presencia de aire, el producto puro comienza a descomponerse después de un día, dando lugar a $\mathrm{SiO}_{2}$. A finales de los años sesenta se llevó a cabo la amonólisis del fenil-metoxi o etoxi-dicloro-silano, encontrando la formación de una mezcla de productos desde los cuales se aislaron el fenil-amino-dimetoxi o dietoxi-silanos y fenilmetoxi o etoxi-silazanos. (Andrianov et al., 1967) Los amino-silanos condensaron por acción de la temperatura (calentamiento a $180-190{ }^{\circ} \mathrm{C}$ ) liberando amoniaco. Finalmente, a mediados de los años ochenta se compilaron los resultados y metodologías de estas investigaciones previas y se patentó la síntesis generalizada de organil-amino-alcoxi-silanos (Arkles et al., 1985).

El uso de amiduros de magnesio es mucho menos extendido y la mayoría de reportes son patentes que, como era de esperarse, describen los procedimientos y reactivos de manera muy general. Esta ruta involucra la formación del amiduro a partir de una reacción de intercambio entre un reactivo de Grignard y la amina, y su posterior interacción con el alcoxi-silano. Como ejemplos de esta ruta tenemos la preparación de di(aminocíclo)-dialcoxi-silanos (Ikai et al., 1999) y alquilamino-trietoxi-silanos (Yoshikiyo et al., 2004). En ambos trabajos los tetraalcoxi-silanos de partida reaccionan con el amiduro en una mezcla de solventes constituida por un éter y un hidrocarburo. De acuerdo con los autores, una de las ventajas de utilizar la mezcla es que se mantienen aislados el compuesto buscado y la sal.

En este trabajo se implementaron dos metodologías para sintetizar tres fenil-amino-dietoxisilanos a partir del fenil-trietoxi-silano. Una de ellas 
involucra cloración y condensación con dietilamina, dipropilamina o n-metilbutilamina. La otra utiliza una reacción directa del silano de partida con bases de amiduros de magnesio, preparados a partir de las tres aminas mencionadas anteriormente. Se realizó un seguimiento cromatográfico de la estabilidad y el comportamiento de descomposición de los productos. Los resultados permitieron establecer bajo las mejores condiciones para obtener productos estables útiles como precursores de materiales híbridos.

\section{Sección experimental}

\subsection{Materiales y equipos}

Todas las reacciones fueron llevadas a cabo bajo atmosfera inerte usando vidriería estándar Schlenk. El fenil-trietoxi-silano (FTES, pureza > 98\%), el tetraetoxi-silano (TEOS, pureza > 99\%) y el cloruro de isopropilmagnesio (solución $\sim 1,3 \mathrm{M}$ en THF), fueron comprados a Sigma-Aldrich y utilizados sin tratamiento previo. Los reactivos trietilamina (TEA), dietilamina (DEA), dipropilamina (DPA), tolueno (emplura), tetrahidrofurano (THF, emplura), $\mathrm{N}$-metil-butilamina (MBA), tricloruro de aluminio, cloruro de butanoilo y el cloroformo deuterado tienen purezas $>98 \%$ y fueron comprados a químicos Merck. Las aminas fueron destiladas desde hidróxido de potasio y los solventes desde Na y mantenidos en malla molecular 4-Å hasta su uso. Los análisis de resonancia magnética nuclear protónicos fueron realizados en un Bruker Fourier de $300 \mathrm{MHZ}$ usando cloroformo deuterado como solvente. Los cromatogramas y espectros de masas fueron obtenidos en un equipo shimadzu GCMS QP2010 Ultra, con una columna capilar tipo DB-5. Se inyectó $1 \mu \mathrm{L}$ de muestra usando helio como gas de arrastre a una velocidad de 1,2 mL/min, en la modalidad Split (relación de 100:1). La temperatura en el puerto de inyección fue de $250{ }^{\circ} \mathrm{C}$ y para el horno se utilizó el siguiente programa: temperatura inicial $80^{\circ} \mathrm{C}$ por 4 minutos, luego un incremento de $10^{\circ} \mathrm{C}$ por minuto hasta 300 ${ }^{\circ} \mathrm{C}$, manteniendo esta temperatura por 4 minutos.
2.2. Síntesis de amino-silanos a partir del fenil-trietoxi-silano (ruta 1)

En una primera etapa se prepara el fenildietoxi-cloro-silano a partir de fenil-trietoxi-silano mediante una adaptación de una metodología reportada en la literatura (Mirskov et al., 2008). En un tubo de polipropileno se añade el trialcoxi-silano (10 mmol; 2,4 mL) y una mezcla que contiene $\mathrm{AlCl}_{3}$ $(0,019 \mathrm{mmol} ; 2,9 \mathrm{mg})$ y cloruro de butanoilo (20 mmoL; 2,0 mL). Se desplaza el aire con Argón y se sella herméticamente el tubo permitiendo reacción con agitación magnética a $80{ }^{\circ} \mathrm{C}$ por 1,5 horas. El producto de la reacción es transferido a un balón de fondo redondo de $25 \mathrm{~mL} \mathrm{y}$, con el fin de eliminar los componentes volátiles, se destila al vacío a $80^{\circ} \mathrm{C}$ durante 25 minutos. En una segunda etapa, y después de regenerar la atmosfera inerte, se adicionan al cloro-silano $15 \mathrm{~mL}$ de solvente (tolueno) y lentamente una mezcla que contiene la amina secundaria (10 mmoL que equivalen a 1,0 $\mathrm{mL}$ de DEA; $1,4 \mathrm{~mL}$ de DPA o $1,2 \mathrm{~mL}$ de MBA) y trietilamina (10 mmol; $1,4 \mathrm{~mL}$ ) disueltas en $5 \mathrm{~mL}$ del solvente. Se permitió reacción bajo agitación magnética por 2 horas a temperatura ambiente.

\subsection{Síntesis de amino-silanos} usando amiduros de magnesio (ruta 2)

En un tubo de polipropileno se añaden 2,5 mL de tolueno; 0,25 mL de THF y 5,0 o $10 \mathrm{mmol}$ de la amina secundaria (DEA, DPA o MBA). A la mezcla se le adiciona lentamente 5,0 o $10 \mathrm{mmol}$ de cloruro de isopropilmagnesio (tomados a partir de una solución $\sim 1,3$ M en THF). Se permite la reacción de intercambio de Grignard por una hora bajo agitación magnética y se incorpora gota a gota el feniltrietoxi-silano (2,5 mmol; 0,62 $\mathrm{mL})$. El sistema se lleva a $40{ }^{\circ} \mathrm{C}$ y se permite reacción por 2 horas.

\subsection{Purificación, caracterización} y seguimiento de la estabilidad

Con el fin de remover la mayor cantidad de sólido que se forma en las reacciones de las secciones 
2.2 y 2.3 (cloruro de trietilamonio o etoxi cloruro de magnesio respectivamente), la mezcla es centrifugada a $0{ }^{\circ} \mathrm{C}$ y $3500 \mathrm{rpm}$ durante 15 minutos y el sobrenadante, aún frio, es pasado a través de papel filtro con porosidad de $0,45 \mu \mathrm{m}$. El filtrado puede ser: a) diluido en tolueno y caracterizado inmediatamente usando cromatografía de gases acoplada a masas (CG-MS), b) almacenado para hacer un seguimiento por CG-MS, de la estabilidad de las moléculas con el tiempo o c) pre-concentrado para eliminar el solvente y destilado entre $10-20 \mathrm{mmHg}$ para intentar obtener el silano puro. El destilado es caracterizado usando resonancia magnética nuclear protónica $\left({ }^{1} \mathrm{H}\right.$ RMN). En los análisis cromatográficos de los numerales a) y b) se inyectó $1 \mu \mathrm{L}$ de una dilución de 100 $\mu \mathrm{L}$ del filtrado en $1500 \mu \mathrm{L}$ de tolueno. La estabilidad fue monitoreada a partir del día de la síntesis y durante dos semanas.

\section{Resultados y discusión}

Los cromatogramas de los filtrados de las rutas 1 y 2, presentados en la Figura 3, indican que ambas estrategias de síntesis proceden hasta que gran parte del FTES (señal 1) se ha consumido. En las reacciones se produce exclusivamente fenil-dietilamino (señal 2), fenil-dipropilamino (señal 3) o fenil-metilbutilamino (señal 4) -dietoxi-silano, con rendimientos que varían entre el 91 y el 98\%. Los espectros de masas, asociados a cada señal cromatográfica, son presentados en la parte inferior de la figura. El pico base, originado por la ruptura homolítica del enlace C-C en posición alfa con respecto al nitrógeno, fue utilizado para la identificación del amino-silano. De manera que los fragmentos con $\mathrm{m} / \mathrm{z}=252,2 ; 266,2$ y $238,1 \mathrm{~g} / \mathrm{mol}$, observados para las señales 2,3 y 4 respectivamente, corresponden a los iones moleculares $\left(\mathrm{C}_{6} \mathrm{H}_{5}\right)\left(\mathrm{CH}_{2} \mathrm{CH}_{3} \mathrm{O}\right)_{2} \mathrm{Si}\left(\mathrm{N}+\left(=\mathrm{CH}_{2}\right)(\mathrm{Z})\right)$, con $\mathrm{Z}=\mathrm{CH}_{2} \mathrm{CH}_{3}, \mathrm{CH}_{2} \mathrm{CH}_{2} \mathrm{CH}_{3}$ o $\mathrm{CH}_{3}$.

Figura 3. Ejemplo típico de los cromatogramas de los filtrados que son obtenidos por las rutas 1 y 2 . La señal con el número 1 corresponde a FTES que no reacciona, las señales marcadas con 2, 3 y 4 corresponden al fenil-dietilamino, fenil-metilbutilamino y fenil-dipropilamino-dietoxi-silano respectivamente. En la parte inferior de la figura se presentan los espectros de masas para cada señal. El pico base para los amino-silanos es la ruptura homolítica del enlace C-C en posición alfa con respecto al nitrógeno
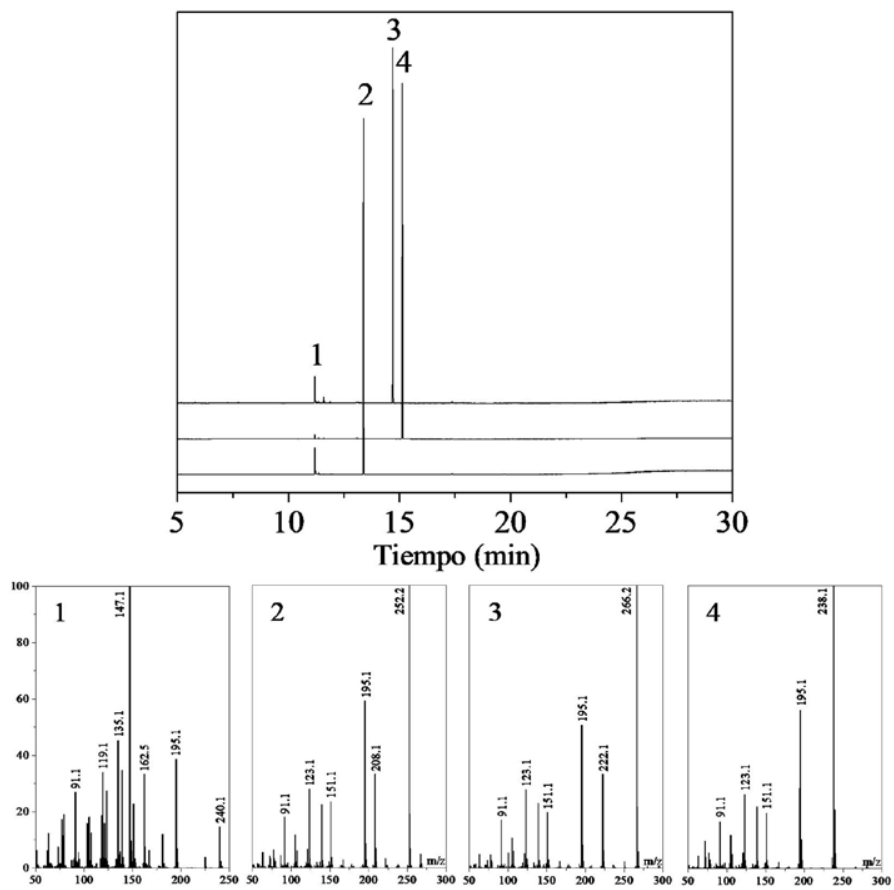
Figura 4. Cromatogramas de los filtrados para el dipropilamino-trietoxi y el fenil-dipropilamino-dietoxi-silano obtenidos usando amiduros de magnesio y TEOS o FTES con una relación 4:1

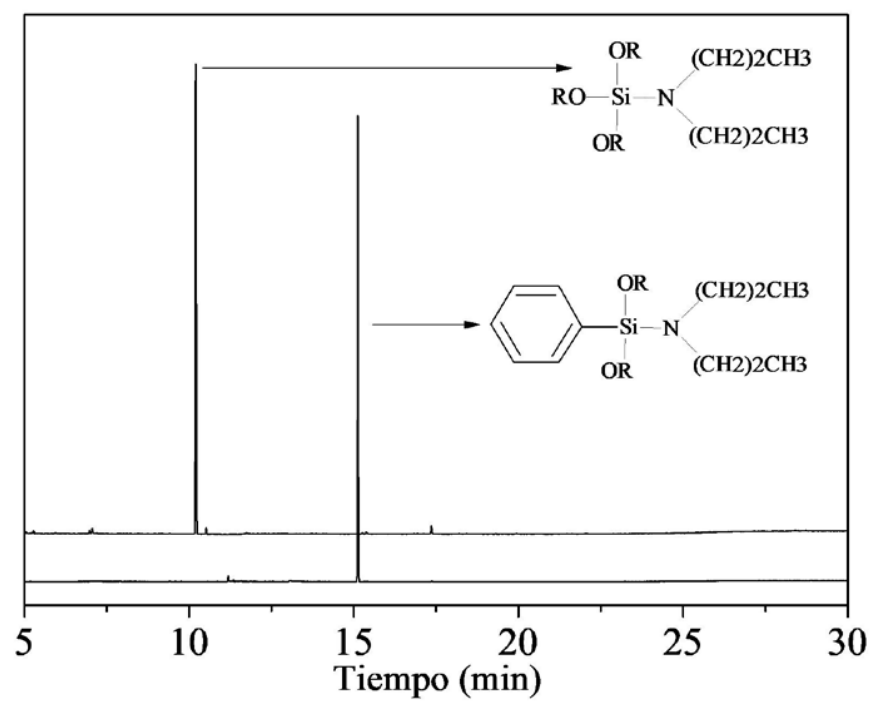

Aunque en las reacciones se utilizaron relaciones molares cloruro de butanoilo:FTES (ruta 1) o amiduro:FTES (ruta 2) de 2:1, sólo se reemplaza un grupo etoxi. Este comportamiento contrasta con la patente de Ikai y colaboradores (1999), en donde se afirma que al utilizar amiduros preparados a partir de aminas cíclicas en una relación 2:1, se pueden sustituir eficientemente dos grupos alcoxi. Con el fin de corroborar nuestro resultado, se realizaron dos síntesis adicionales usando el amiduro preparado a partir de la dipropilamina y FTES o TEOS en una relación 4:1. En ningún caso se logró la disustitución (ver Figura 4).

Por otro lado, las condiciones experimentales utilizadas en la destilación dieron lugar a un proceso de purificación no reproducible, independientemente de la ruta de síntesis implementada. Aunque en algunos casos se logró obtener el silano (ver Figura 5), en la mayoría de las oportunidades el filtrado polimerizó durante el calentamiento, dando lugar a sólidos amorfos que corresponden a silsesquioxanos. También se observó un aumento gradual en la viscosidad de los destilados en un periodo de tiempo inferior a 24 horas, evidenciando su inestabilidad bajo condiciones atmosféricas.
Figura 5. 'H RMN para el fenil-dietilamino-dietoxi-silano obtenido por la ruta 2 (espectro A) y el fenil-dipropil-dietoxi-silano obtenido por ruta 1 (espectro B)

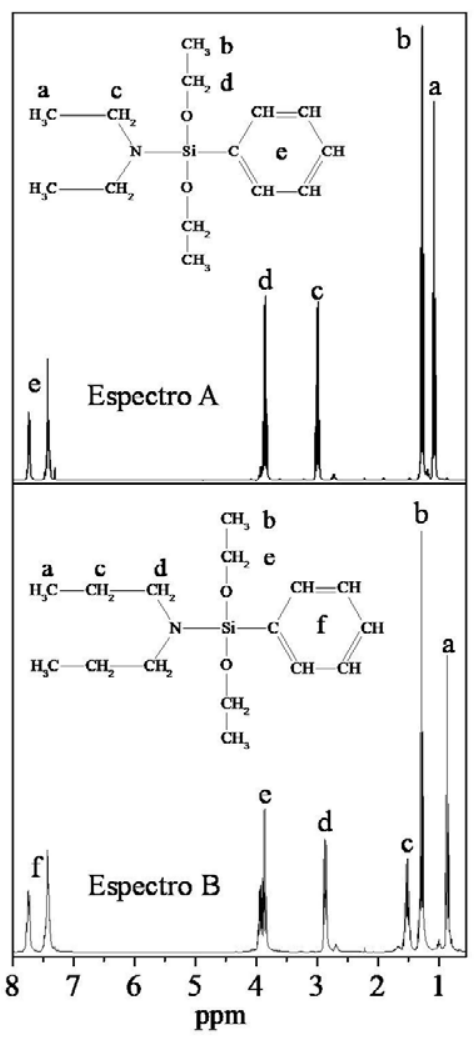


Figura 6. Efecto del tiempo sobre el proceso de descomposición del fenil-dietilamino-dietoxi-silano obtenido por la ruta 1 y por la ruta 2

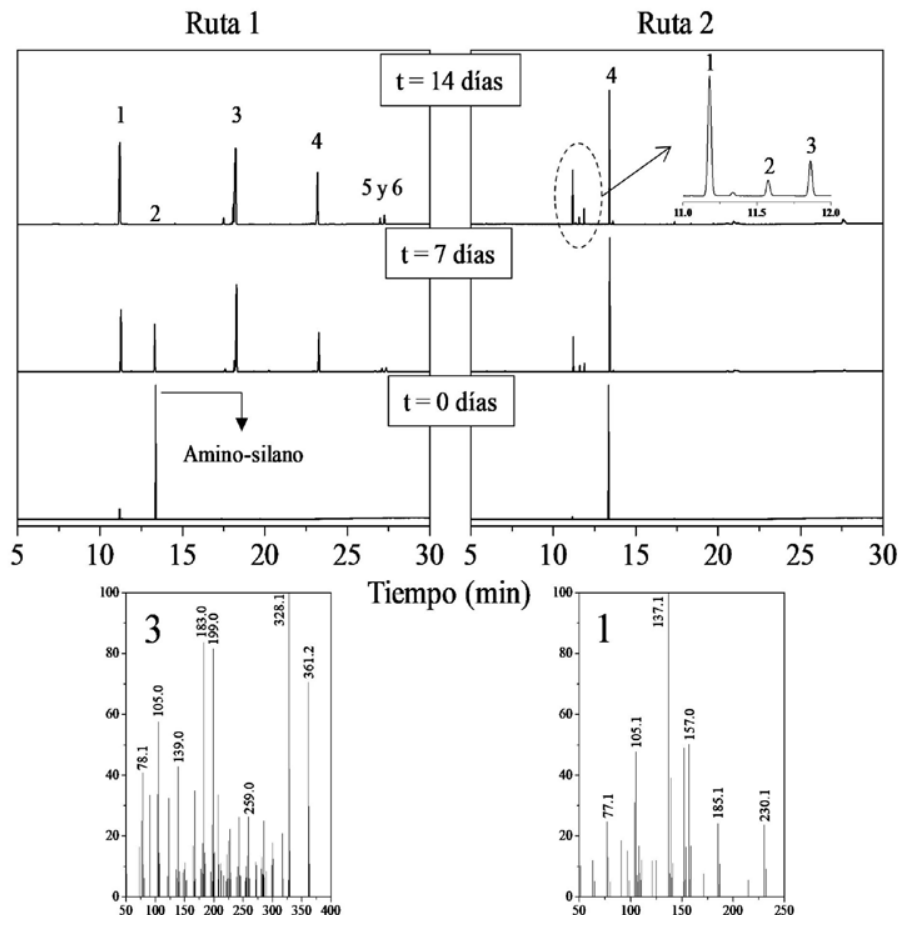

La posibilidad de descomposición durante la purificación por destilación y la labilidad de los destilados son consecuentes con los reportes de Rosnati (1948) y Andrianov (1967). Buscando alternativas para la preservación de los tres amino-silanos, se realizó un seguimiento de su estabilidad en el solvente de reacción obtenido después de la centrifugación y el filtrado. La Figura 6 presenta los cromatogramas obtenidos durante este seguimiento para el caso particular del fenil-dietilamino-dietoxisilano. El mismo comportamiento fue observado con las moléculas de fenil-dipropilamino- y fenilmetilbutilamino-dietoxi-silano.

Es claro en la figura que la estabilidad y el mecanismo de descomposición son fuertemente dependientes de la ruta de síntesis. Se pudo establecer que los amino-silanos obtenidos por la ruta 1 , se descomponen por completo en un periodo de dos semanas, dando lugar a especies de mayor peso molecular (ver por ejemplo el espectro de masas para la señal 3). Los compuestos obtenidos por la ruta 2 son mucho más estables en el filtrado y su descomposición da lugar a especies de menor peso (ver por ejemplo el espectro para la señal 1). Para el caso particular del fenil-dietilamino-dietoxi-silano (señal 4, ruta 2), se observa que después de 14 días aun permanece más del $60 \%$ de su concentración inicial. El origen de esta diferencia puede ser atribuido a la naturaleza química de las sales obtenidas como subproducto. Estos sólidos no pueden ser removidos en su totalidad durante la filtración y en la fase líquida permanecen trazas residuales (Stueger et al., 2010). En presencia de la humedad del aire, la sal obtenida en la ruta $1\left(\mathrm{NH}\left(\mathrm{CH}_{2} \mathrm{CH}_{3}\right)_{3} \mathrm{Cl}\right)$ actúa como un catalizador ácido, promoviendo hidrólisis y formación de enlaces siloxano. Dando lugar a las especies de mayor peso. Esto no sucede con el $\mathrm{CH}_{3} \mathrm{CH}_{2} \mathrm{OMgCl}$. Un comportamiento semejante fue reportado por otros autores (Stueger et al., 2010), quienes prepararon amino-ciclohexasilanos a partir 
de cloro-silanos y $\mathrm{NH}_{3} \mathrm{O} \mathrm{NaNH}_{2}$, obteniendo como subproductos las sales $\mathrm{NH}_{4} \mathrm{Cl}$ y $\mathrm{NaCl}$ respectivamente. Después de la filtración, ellos observaron cómo sus moléculas condensaron a temperatura ambiente en el primer caso, mientras que permanecieron estables en el segundo.

\section{Conclusiones}

Las dos rutas de síntesis evaluadas son útiles para obtener fenil-amino-dietoxi-silanos con buenos rendimientos. La purificación y almacenamiento de estas moléculas requiere de un cuidado especial, ya que ellas se descomponen fácilmente con la temperatura y la humedad del aire. También se descomponen rápidamente en contacto con las sales ácidas que se forman como subproducto de la síntesis convencional; aquella que involucra condensación de cloro-silanos y aminas. Consecuentemente, si se desea preservar el amino-silano para una aplicación posterior, es mejor prepararlo mediante la reacción directa entre el alcoxi-silano y el amiduro de magnesio. En este caso la sal neutra que se forma no es tan agresiva con el producto. Por otro lado, con las aminas lineales evaluadas, no es posible sustituir más de un grupo etoxi del reactivo de partida, independientemente de la relación molar utilizada.

\section{Agradecimientos}

Los autores agradecen a Colciencias y a la Universidad de Antioquia por el soporte financiero para la realización de este trabajo (proyecto con código 111552128184).

\section{Referencias}

Abe, Y. y Gunji, T., 2004. Oligo- and polysiloxanes. Progress in Polymer Science, 29(3), pp. 149-182.

Andrianov, K. A., Kotrelev, G. V., Lavrukhin, B. D. y Orobinskaya, L. N., 1967. Organoalkoxycyclosilazanes. Bulletin of the Academy of Sciences of the USSR, Division of chemical science, 16(10), pp. 2179-2184.

Arkles, B. C., Peterson, W. R. y Rokowski, J. M., Google Patents, 1985. Mixed alkoxyaminosilanes, methods of making same and vulcanizing silicons prepared therefrom. US4491669 A.

Bauer, J. O. y Strohmann, C., 2012. One-step conversion of methoxysilanes to aminosilanes: a convenient synthetic strategy to N,O-functionalised organosilanes. Chemical Communications, 48(57), pp. 72127214.

Colvin, E. W., 1990. Silicon reagents in organic synthesis. 2nd ed. San Diego: Academic Press Limited.

Drozd, J., 1981. Chemical Derivatization in Gas Chromatography. Amsterdam: Elsevier Scientific Publishing Company.

Ikai, S., Sakakibara, Y. y Fukunaga, T., Google Patents, 1999. Process for preparing di (polycyclic amino) dialkoxysilane. US5939573 A.

Ishibashi, K., Tsue, H., Tokita, S., Matsui, K., Takahashi, H. y Tamura, R., 2006. Regioselectively N-Methylated Azacalix[8]arene Octamethyl Ether Prepared by Catalytic Aryl Amination Reaction Using a Temporal N-Silylation Protocol. Organic Letters, 8(26), pp. 5991-5994.

Marciniec, B., Kostera, S., Wyrzykiewicz, B. y Pawluc, P., 2015. Ruthenium-catalyzed dealkenative N-silylation of amines by substituted vinylsilanes. Dalton Transactions, 44(2), pp. 782-786.

Miner, J. C., Bryan, L., Holysz, J. R. y Pedlow, J. G., 1947. Alkoxyaminosilanes. Industrial \& Engineering Chemistry, 39(11), pp. 1368-1371.

Mirskov, R. G., Rakhlin, V. I., Adamovich, S. N. y Voronkov, M. G., 2008. High-purity alkoxychlorosilanes as new precursors for precipitation of silica. Doklady Chemistry, 421(2), pp. 194-196.

Molnár, B., Fodor, B., Boldizsár, I. y Molnár-Perl, I., 2015. Quantitative Silylation Speciations of Primary Phenylalkyl Amines, Including Amphetamine and 3,4-Methylenedioxyamphetamine Prior to Their Analysis by GC/MS. Analytical Chemistry, 87(20), pp. 10188-10192.

Rosnati, L., 1948. Derivatives of chlorosilanes. I. Amines Gazz. chim. ital., 78(pp.

Spence, D. P., Pearlstein, R. M., Lei, X., Xiao, M., Ho, R., O'neill, M. L. y Chandra, H., Google Patents, 2013. Volatile monoamino-dialkoxysilanes and their use for creating silicon-containing films. EP2620440 A1.

Stueger, H., Fuerpass, G., Mitterfellner, T. y Baumgartner, J., 2010. Synthesis and Reactivity of NH2 Derivatives 
of Dodecamethylcyclohexasilane. Organometallics, 29(3), pp. 618-623.

Yoshikiyo, M., Fukunaga, T., Sato, H., Machida, T., Ikeuchi, H., Yano, T. y Tanaka, Y., Google Patents, 2004. Catalysts for polymerization or copolymerization of $\alpha$-olefins, catalyst components thereof, and processes for polymerization of $\alpha$-olefins with the catalysts. US20050202958 A1. 